\title{
TGF- $\beta 1$ and IGF-1 influence the re-differentiation capacity of human chondrocytes in 3D pellet cultures in relation to different oxygen concentrations
}

\author{
ANIKA JONITZ, KATRIN LOCHNER, THOMAS TISCHER, DORIS HANSMANN and RAINER BADER \\ Department of Orthopaedics, Biomechanics and Implant Technology Research Laboratory, \\ University Medicine Rostock, Rostock, Germany
}

Received April 10, 2012; Accepted June 5, 2012

DOI: $10.3892 /$ ijmm.2012.1042

\begin{abstract}
To prevent de-differentiation of chondrocytes in vitro, the $3 \mathrm{D}$ environment, growth factors and different oxygen concentrations were considered. In this in vitro study, we quantified the influence of insulin-like growth factor (IGF)-1 and/or transforming growth factor (TGF)- $\beta 1$ under differing oxygen $\left(5 / 21 \% \mathrm{O}_{2}\right)$ levels on the proliferation and synthesis rates of hyaline extracellular matrix (ECM) components in chondrogenic pellet cultures. Human chondrocytes isolated from articular cartilage were transferred into conical tubes to form pellets. Pellets were stimulated with TGF- $\beta 1$ and/or IGF-1. After 2 and 5 weeks of cultivation the DNA concentration and expression of pro-collagen type 1, type 2 and aggrecan were analysed. Under hypoxia the DNA content remained stable. In contrast, under normoxia, cells showed an increase of DNA concentration after stimulation with TGF- $\beta 1 /$ IGF- 1 and TGF- $\beta 1$. Nevertheless, DNA contents under normoxia did not reach the values of hypoxic-cultivated cells. Under both culture conditions a reduced synthesis of pro-collagen type 1 could be determined. Although the expression of pro-collagen type 2 was significantly higher under normoxia, a decrease in the case of TGF- $\beta 1 /$ IGF-1- and IGF-1-stimulated cells was observed. Under hypoxia pro-collagen type 2 contents remained stable or increased for TGF- $\beta 1 /$ IGF-1-stimulated cells. Furthermore, incubation with growth factors resulted in aggrecan accumulation under hypoxia, while a reduced expression under normoxia could be determined for TGF- $\beta 1 /$ IGF-1- and IGF-1-stimulated cells. Our results demonstrate that the treatment with growth factors causes differences in the expression of ECM compounds within pellet cultures. While under normoxia TGF- $\beta 1$ alone leads to a positive effect of the expression of hyaline cartilage-
\end{abstract}

Correspondence to: Anika Jonitz, Department of Orthopaedics, Biomechanics and Implant Technology Research Laboratory, University Medicine Rostock, Doberaner Strasse 142, D-18057 Rostock, Germany E-mail: anika.jonitz@med.uni-rostock.de

Key words: cartilage, chondrocyte, extracellular matrix, growth factors, oxygen, pellet culture specific ECM components, an additive effect of both growth factors was only determined under hypoxia.

\section{Introduction}

Hyaline cartilage is characterised by avascularity, a rich extracellular matrix (ECM), low cell density and turnover. As a consequence, the capacity of cartilage regeneration is considerably reduced compared to other connective tissues. As a result, traumatic cartilage defects (1) and degenerative changes as a consequence of the rising age (2) are associated with joint pain and limited functions (3) and ultimately lead to osteoarthritis. Therefore, the major challenge of a successful treatment of cartilage defects in orthopaedic surgery is to prevent this progressive degeneration.

Autologous chondrocyte transplantation (ACT) is a possible technique for cartilage treatment with promising results (4). For this method, the patient's own chondrocytes need to be isolated and afterward cultivated in vitro to increase cell number. Hereby, proliferation of cells is associated with a de-differentiation process towards a fibroblastoid phenotype. As a result, chondrocytes lose their differentiated characteristics like collagen type 2 and proteoglycan synthesis (5). In order to synthesise hyaline ECM components, cells have to re-differentiate into the chondrocytic phenotype. For cartilage regeneration, it is well known that a three-dimensional (3D) environment could support re-differentiation processes of chondrocytes $(6,7)$. Furthermore, essential chondrogenic growth factors were identified which could also support differentiation processes in vitro. Hereby, growth factors are stored in the ECM and will be released under specific conditions (e.g. surface damaging) to regulate cellular behaviour (8).

One important growth factor in cartilage is the insulinlike growth factor (IGF)-1 which is the major anabolic factor for stimulating matrix synthesis by working in an autocrine and paracrine way (9). IGF-1 effects and therefore the biological function are modulated by the interaction of IGF-binding proteins (IGFBP). Due to the binding of IGF-1 to IGFBP, the transport and also the availability of IGF-1 to chondrocytes are regulated (8).

Beside IGF-1, transforming growth factor (TGF)- $\beta 1$ is also responsible for matrix synthesis by increasing the expression of 
collagen type 2 and accumulating proteoglycans (10). However, effects of TGF- $\beta 1$ on chondrocytes are dependent on the conditions of the target cells. TGF- $\beta 1$ could stimulate as well as inhibit cell proliferation and proteoglycan synthesis (11). It is well described that the combination of TGF- $\beta 1$ and IGF- 1 causes an induction of collagen type 2 mRNA in de-differentiated chondrocytes derived from articular cartilage (12). Furthermore, in alginate-encapsulated de-differentiated chondrocytes the treatment of IGF- 1 and TGF- $\beta 1$ resulted in an accumulation of ECM components like collagen type 2, glycosaminoglycan (GAG) and aggrecan $(13,14)$.

The aim of this study was to analyse the effects of TGF- $\beta 1$, IGF-1 and the combination of both on the proliferation and differentiation capacity of human chondrocytes in pellet cultures under different oxygen concentrations. Hereby, we investigated which conditions could have a positive effect on chondrogenic re-differentiation and therefore accumulation of ECM.

For the experiments we used cells derived from osteoarthritic $(\mathrm{OA})$ human cartilage which is characterised by limited reactions onto IGF-1 and TGF- $\beta$ (15). One possible reason could be the increased oxidative stress and therefore the enrichment of reactive oxygen species (ROS) which lead to a reduced effect of IGF-1 (2). Therefore, we hypothesise that the reduction of the oxygen content from $21 \%$ (normoxia) to $5 \%$ (hypoxia) during cultivation could influence the effects of IGF-1 and TGF- $\beta 1$ positively because of a decreased oxidative stress for cells.

\section{Materials und methods}

Chondrocyte isolation and cultivation. Articular knee cartilage was obtained from 12 patients (4 male donors, mean age, $71 \pm 12.4$ years; 8 female donors, mean age, $69.8 \pm 6.8$ years) undergoing primary knee replacement. The samples were collected after obtaining consent from the patients and approval by the Local Ethics Committee (registration no. A2009 17).

For isolation of primary chondrocytes the cartilage was removed from the underlying bone, cut into small pieces and washed three times in PBS (PAA Laboratories, Coelbe, Germany). Afterwards the cartilage $(\sim 3 \mathrm{~g} \mathrm{w} / \mathrm{w})$ was treated with $1 \%$ Gibco $^{\circledR}$ Trypsin/EDTA (Invitrogen, Darmstadt, Germany) for $20 \mathrm{~min}$ at $37^{\circ} \mathrm{C}$ followed by $0.2 \%$ collagenase A (Roche Diagnostics GmbH, Mannheim, Germany) in DMEM supplemented with $10 \%$ FCS, $1 \%$ amphotericin B and $1 \%$ Gibco ${ }^{\circledR}$ penicillin/streptomycin (Invitrogen) at $37^{\circ} \mathrm{C}$ overnight. The cell suspension was filtered through a cell strainer (pore size, $70 \mu \mathrm{m}$; Nunc, Wiesbaden, Germany) and centrifuged at $118 \mathrm{x}$ g for $10 \mathrm{~min}$. The cell pellet was resuspended in DMEM with the supplements mentioned above and ascorbic acid.

Freshly isolated chondrocytes were plated in a $25-\mathrm{cm}^{2}$ culture flask with $8 \mathrm{ml}$ culture medium with supplements and incubated in a humidified atmosphere at $37^{\circ} \mathrm{C}$ and $5 \% \mathrm{CO}_{2}$. The medium was changed every second day. Cell proliferation was determined visually by microscopic control. When cell confluence reached $90 \%$ ( $\sim 5 \times 10^{5}$ cells $/ 25 \mathrm{~cm}^{2}$ flask), the cells were trypsinised and split at a ratio 1 to 6 . For all experiments cryo-conserved chondrocytes were used. After thawing, cells were centrifugated at $118 \mathrm{x}$ g for $10 \mathrm{~min}$, transferred into $75-\mathrm{cm}^{2}$ flasks (passage 2) and incubated in a humidified
Table I. Three different growth factor combinations in the cell medium.

\begin{tabular}{lccc}
\hline & $\begin{array}{c}\text { Medium } \\
\text { combination 1 }\end{array}$ & $\begin{array}{c}\text { Medium } \\
\text { combination 2 }\end{array}$ & $\begin{array}{c}\text { Medium } \\
\text { combination 3 }\end{array}$ \\
\hline TGF- $\beta 1$ & $50 \mathrm{ng} / \mathrm{ml}$ & $50 \mathrm{ng} / \mathrm{ml}$ & - \\
IGF-1 & $50 \mathrm{ng} / \mathrm{ml}$ & - & $50 \mathrm{ng} / \mathrm{ml}$ \\
\hline
\end{tabular}

atmosphere at $37^{\circ} \mathrm{C}, 5 \% \mathrm{CO}_{2}$ and $5 \% \mathrm{O}_{2}$ (hypoxia) or $21 \% \mathrm{O}_{2}$ (normoxia).

In passage three, chondrocyte pellet cultures with an amount of $5 \times 10^{5}$ cells/pellet were prepared. Therefore, cells were trypsinised, centrifuged at $118 \mathrm{x}$ g for $10 \mathrm{~min}$ and an appropriate aliquot of cells was split in three different medium compositions. Subsequently, the cell suspension was transferred into conical tubes. Pellet cultures were incubated in serum-free DMEM containing ascorbic acid (final concentration, $50 \mu \mathrm{g} / \mathrm{ml}$; Sigma) dexamethasone (final concentration, $100 \mathrm{nM}$; Sigma), ITS ${ }^{\text {TM }}$ (complete medium to ITS ${ }^{\text {TM }}$ in a 100:1 ratio; Becton-Dickinson, Heidelberg, Germany) and the chondrogenic growth factors IGF-1 (R\&D Systems, Wiesbaden, Germany) and/or TGF- $\beta 1$ (Tebu-Bio, Offenbach, Germany) in three different compositions, shown in Table $\mathrm{I}$, at $37^{\circ} \mathrm{C}$ in a humidified atmosphere of $5 \% \mathrm{CO}_{2}$ and the appropriate oxygen concentration for 5 weeks. The culture medium was also changed every second day.

DNA isolation and quantification. For the DNA isolation pellet cultures of each stimulating experiment were transferred in $2 \mathrm{ml}$ homogenisation tubes containing small steel beads (Precellys-Steel kit, $2.8 \mathrm{~mm}$; PeqLab Biotechnologie $\mathrm{GmbH}$, Erlangen, Germany), covered with $100 \mu 1$ TE-buffer and homogenised for $30 \mathrm{sec}$ at $5,000 \mathrm{xg}$.

DNA isolation was performed using the peqGOLD Tissue DNA mini kit (PeqLab Biotechnologie $\mathrm{GmbH}$ ) according to the manufacturer's instructions. Afterwards, DNA concentrations were measured with the Qubit Fluorometer according to the manufacturer's instructions (Invitrogen).

Pro-collagen type $1(C I C P)$ and type $2(C P 2)$ quantification. Synthesis of pro-collagen type 1 (Quidel, Marburg, Germany) and type 2 (TECOmedical, Buende, Germany) were determined by ELISA. Hence, supernatants of every medium combination were collected and stored at $-20^{\circ} \mathrm{C}$ after 14 and 35 days of incubation. The assay was performed according to manufacturer's instructions. Absorbance was measured at $450 \mathrm{~nm}$ for the CP2 ELISA and $405 \mathrm{~nm}$ for the CICP ELISA using Opsys MR ${ }^{\mathrm{TM}}$ microplate reader (Dynex Technologies, Denkendorf, Germany). The content of pro-collagen type 1 as well as type 2 was normalised to the DNA content (ng procollagen/ng DNA).

Aggrecan (PG-EASIA) quantification. The PG-EASIA (Invitrogen) is a solid phase enzyme amplified sensitivity immunoassay (EASIA). The background of this assay is the use of an oligoclonal system in which monoclonal antibodies are directed against distinct epitopes of proteoglycan. 

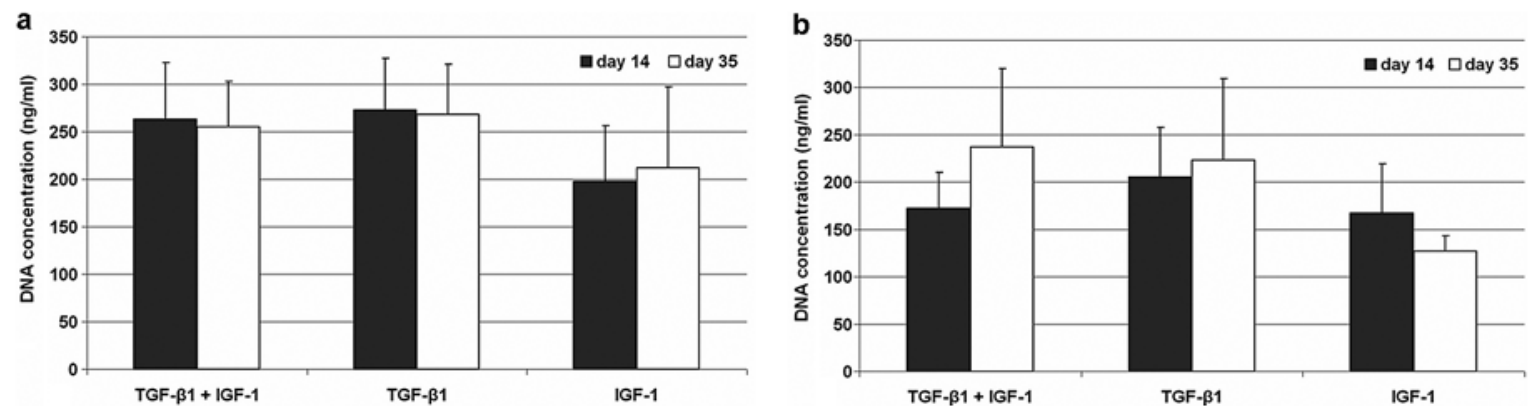

Figure 1. Quantification of DNA content of human chondrocyte pellet cultures under (a) hypoxic and (b) normoxic culture conditions on Day 14 and 35 . Data are presented as mean value \pm standard deviation $(n=3)$. Statistical significance between the two time points was calculated with the Wilcoxon test. Comparison between the three growth factor combinations was calculated with the Kruskal-Wallis test for independent samples. Comparison between the both culture conditions was calculated with the Mann-Whitney test.

After 14 or 35 days of incubation, supernatants of every medium combination were collected and stored at $-20^{\circ} \mathrm{C}$. The assay was performed according to manufacturer's instructions. Absorbance was measured at $450 \mathrm{~nm}$ using the Opsys $\mathrm{MR}^{\mathrm{TM}}$ microplate reader (Dynex Technologies). The content of aggrecan was normalised to the DNA content (ng aggrecan/ ng DNA).

Immunohistochemistry of pellet cultures. For immunohistological examinations of type 2 collagen and aggrecan after 35 days of incubation, pellets were fixed in $4 \%$ formaline (Merck, Darmstadt, Germany), embedded in paraffin and sectioned to $7 \mu \mathrm{m}$ slices.

For immunohistochemistry of collagen type 2, pellet cultures were digested with pronase (Roche) in PBS buffer and hyaluronidase (Sigma) in PBS buffer containing 0.01\% $\mathrm{BSA}$ at $37^{\circ} \mathrm{C}$ for $30 \mathrm{~min}$ in each case to increase the penetration of antibodies into the matrix. Before incubation with the primary antibody, beads were washed in PBS buffer for $5 \mathrm{~min}$ and blocked in PBS buffer containing 5\% goat serum (PAN Biotech, Aidenbach, Germany) for $30 \mathrm{~min}$ at room temperature. Mouse anti-human type 2 collagen antibody (Millipore, Schwalbach/Ts., Germany) was applied at 1:200 dilution in PBS buffer containing $0.1 \%$ goat serum overnight at $4^{\circ} \mathrm{C}$. To detect collagen type 2, Alexa Fluor ${ }^{\circledR} 488$ goat anti-mouse IgG (Invitrogen), diluted 1:200 in PBS buffer, was used for $2 \mathrm{~h}$ at room temperature.

For aggrecan staining, slices of pellet cultures were digested with chondroitinase (Sigma) in $20 \mathrm{mM}$ Tris buffer (Merck) and keratanase (Sigma) in $50 \mathrm{mM}$ Tris buffer at $37^{\circ} \mathrm{C}$ and $5 \% \mathrm{CO}_{2}$ for $30 \mathrm{~min}$. Afterwards, beads were washed in PBS buffer for 5 min and blocked in PBS buffer containing 5\% goat serum. The monoclonal anti-human aggrecan G1-IGD-G2 domain antibody (R\&D Systems), diluted 1:200 in PBS with $1 \%$ goat serum, was incubated overnight at $4^{\circ} \mathrm{C}$. For aggrecan detection, Alexa Fluor ${ }^{\circledR} 488$ goat anti-mouse IgG, diluted 1:200 in PBS containing 1\% goat serum was applied for $1 \mathrm{~h}$ at room temperature. For negative controls, the primary antibody was replaced by PBS buffer and secondary antibody alone.

Afterwards, sections were embedded in Fluoroshield mounting medium containing DAPI (Acris Antibodies GmbH, Herford, Germany) to visualise cell nuclei. Evaluation and documentation of the results were performed using a Nikon Eclipse TS100 microscope with a Nikon Digital Sight DS-2Mv camera. Photographs were acquired and processed with the NIS Elements 3.0 software (Nikon GmbH, Düsseldorf, Germany).

Statistical analysis. Data are presented as means \pm standard deviation. For all analysis human chondrocyte cultures of a minimum of three independent donors were used. Statistical significance between the two time points was calculated with the Wilcoxon test. Comparison between the three medium combinations was calculated with the Kruskal-Wallis test for independent samples or the Mann-Whitney test for paired samples. Comparison between both culture conditions was calculated with the Mann-Whitney test. All statistical analyses were performed with SPSS 15.0 for Windows (SPSS Inc., Chicago, IL, USA). The significance level was determined for $\mathrm{P}<0.05$.

\section{Results}

Chondrocyte pellet culture morphology and proliferation. During incubation of human chondrocytes over a period of 35 days under different growth factor combinations and oxygen concentrations the cell pellets stimulated with IGF-1 showed a compact appearance. This was in contrast to TGF- $\beta 1 /$ IGF-1 and TGF- $\beta 1$ treated cultures, i.e. both pellet cultures were approximately twice as large and showed a rounded to oval appearance. Furthermore, under normoxic conditions chondrocytes of these stimulation experiments showed a higher metabolic activity than cells incubated in IGF-1 alone which was visible by a clear colour change of the medium as a result of acidification. In contrast, under hypoxia pellets of all three stimulating experiments showed an enhanced metabolism during the incubation time.

Under hypoxic conditions DNA contents of all three stimulating experiments remained stable. Cell pellets revealed nearly the same concentration of DNA at both time points after stimulation with TGF- $\beta 1 /$ IGF-1 and with TGF- $\beta 1$. In IGF-1 stimulated pellets a reduced DNA content was observable at both time points compared to the other stimulating experiments. In contrast, normoxic cultivated cells showed an enhanced proliferation after stimulation with TGF- $\beta 1 /$ IGF-1 and TGF- $\beta 1$. Nevertheless, DNA contents under normoxia did not reach the values of hypoxic-cultivated cells. For IGF-1 stimulated pellet cultures a decreased proliferation rate could be observed from Day 14 to 35 (Fig. 1). 

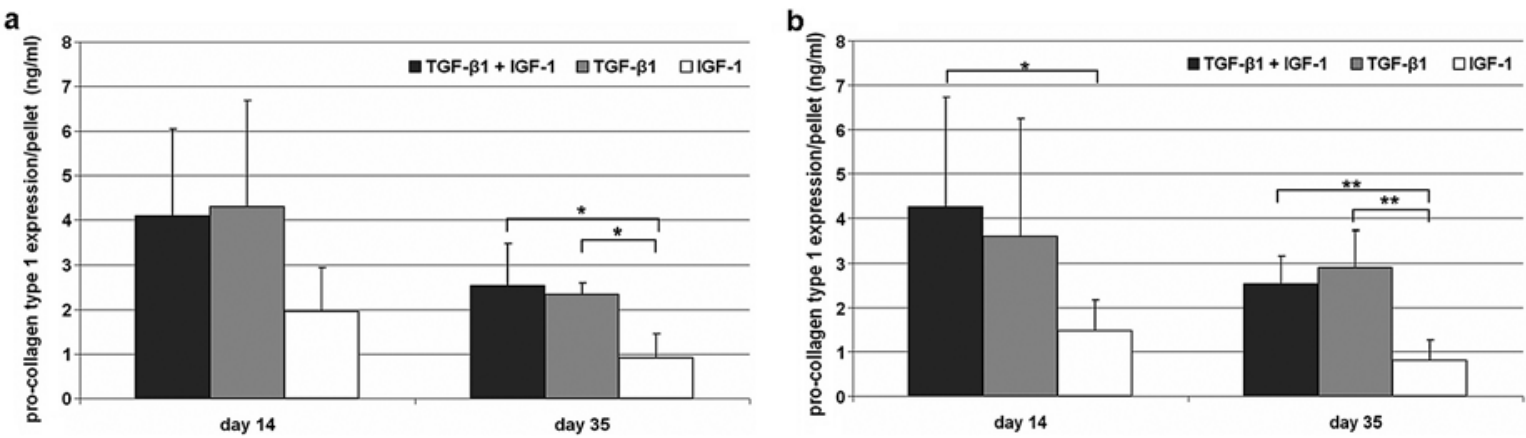

Figure 2. Pro-collagen type 1 synthesis of human chondrocyte pellet cultures under (a) hypoxic and (b) normoxic culture conditions with the different stimulating combinations $(\mathrm{n} \geq 4$, in duplicate). Data are presented as means \pm standard deviation. Comparison between the three growth factor combinations was calculated with the Kruskal-Wallis test for independent samples or the Mann-Whitney test for paired samples; ${ }^{*} \mathrm{P}<0.05$; ${ }^{* *} \mathrm{P}<0.01$. The comparison between the culture conditions was conducted with the Mann-Whitney test. Statistical significance between the two time points was calculated with the Wilcoxon test.

a

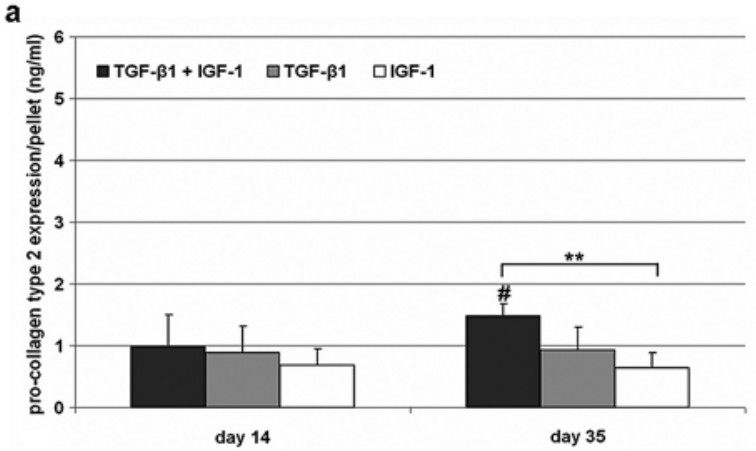

b

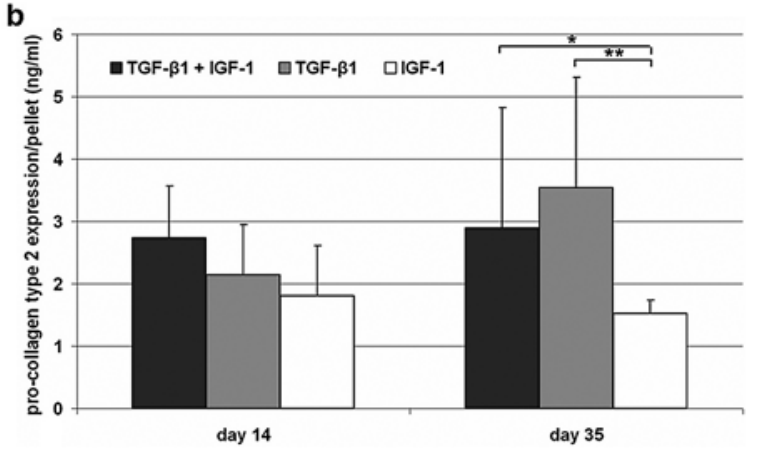

Figure 3. Pro-collagen type 2 synthesis of human chondrocyte pellet cultures under (a) hypoxic and (b) normoxic culture conditions with different stimulating combinations ( $\mathrm{n} \geq 5$, in duplicates). Data are presented as means \pm standard deviation. Comparison between the three growth factor combinations was calculated with the Kruskal-Wallis test for independent samples or the Mann-Whitney test for paired samples; ${ }^{*} \mathrm{P}<0.05 ;{ }^{* *} \mathrm{P}<0.01$. Differences between the culture conditions were assessed with the Mann-Whitney test. Statistical significance between the two time points was calculated with the Wilcoxon test; ${ }^{\prime} \mathrm{P}<0.05$.

Expression of pro-collagen type 1. Synthesis of pro-collagen type 1 declined during the incubation time under hypoxic as well as under normoxic cell culture conditions. Additionally, no significant differences could be determined between both culture conditions. Under hypoxic conditions similar synthesis rates for TGF- $\beta 1 /$ IGF-1- as well as TGF- $\beta 1$-stimulation (Fig. 2) after 14 and 35 days were observable. After IGF-1 stimulation, reduced contents of pro-collagen type 1 were determined compared to the other stimulating experiments. Under normoxia the expression was significantly reduced $(\mathrm{P}=0.04)$ on Day 14 compared to TGF- $\beta 1 / \mathrm{IGF}-1$-treated cells. Nevertheless, after 35 days of incubation significant differences between IGF-1 and the other growth factor combinations were visible for both culture conditions (hypoxia, $\mathrm{P}=0.029$; normoxia, $\mathrm{P}=0.002$ ).

Expression of pro-collagen type 2. Expression of pro-collagen type 2 revealed significant differences between hypoxia and normoxia at both time points [TGF- $\beta 1 / \mathrm{IGF}-1, \mathrm{P}=0.017$ (Day 14); TGF- $\beta 1, \mathrm{P}=0.03$ (Day 14), $\mathrm{P}=0.004$ (Day 35); IGF-1, $\mathrm{P}=0.017$ (Day 14), $\mathrm{P}=0.004$ (Day 35)]. After 14 days of stimulation, hypoxically cultivated cells showed a decreased but similar type 2 pro-collagen content after treatment in all stimulating experiments compared to normoxic cultivated cells (Fig. 3). On Day 35, a significantly $(\mathrm{P}=0.043)$ increased synthesis rate of pro-collagen type 2 could be determined after TGF- $\beta 1 /$ IGF-1 stimulation under hypoxia. In contrast, the content of pro-collagen type 2 remained stable after sole stimulation with TGF- $\beta 1$ or IGF-1.

Under normoxic culture conditions significant differences between IGF-1 and TGF- $\beta 1 /$ IGF-1 $(\mathrm{P}=0.026)$ as well as IGF-1 and TGF- $\beta 1(\mathrm{P}=0.002)$ stimulated pellets were detected after 35 days. Additionally, an increased synthesis rate could only be shown after single treatment with TGF- $\beta 1$ (Fig. 3).

Collagen type 2 staining resulted in marginal differences between hypoxia and normoxia. Compared to TGF- $\beta 1 /$ IGF-1 and TGF- $\beta 1$ treatments, IGF- 1 stimulated pellets showed a less pronounced staining under both culture conditions (Fig. 4a-f).

Synthesis of aggrecan. Under hypoxic culture conditions the synthesis of aggrecan resulted in a distinct but non-significant accumulation of the protein content from Day 14 to 35 . At both time points, significant differences could be determined between IGF-1 stimulated cells and TGF- $\beta 1 / \mathrm{IGF}-1$ as well as TGF- $\beta 1$ stimulated ones $(\mathrm{P}=0.029)$. Furthermore, on Day 35, more aggrecan was synthesised by cells stimulated with the combination of the growth factors TGF- $\beta 1$ and IGF- 1 compared to the single stimulating experiments. In contrast to hypoxic conditions on Day 35, an accumulation of aggrecan was detectable for TGF- $\beta$-1 stimulated cells only. Additionally, normoxic cultivated cells showed a reduced expression of aggrecan from Day 14 to 35 after stimulation with TGF- $\beta 1 /$ IGF-1. Significant 

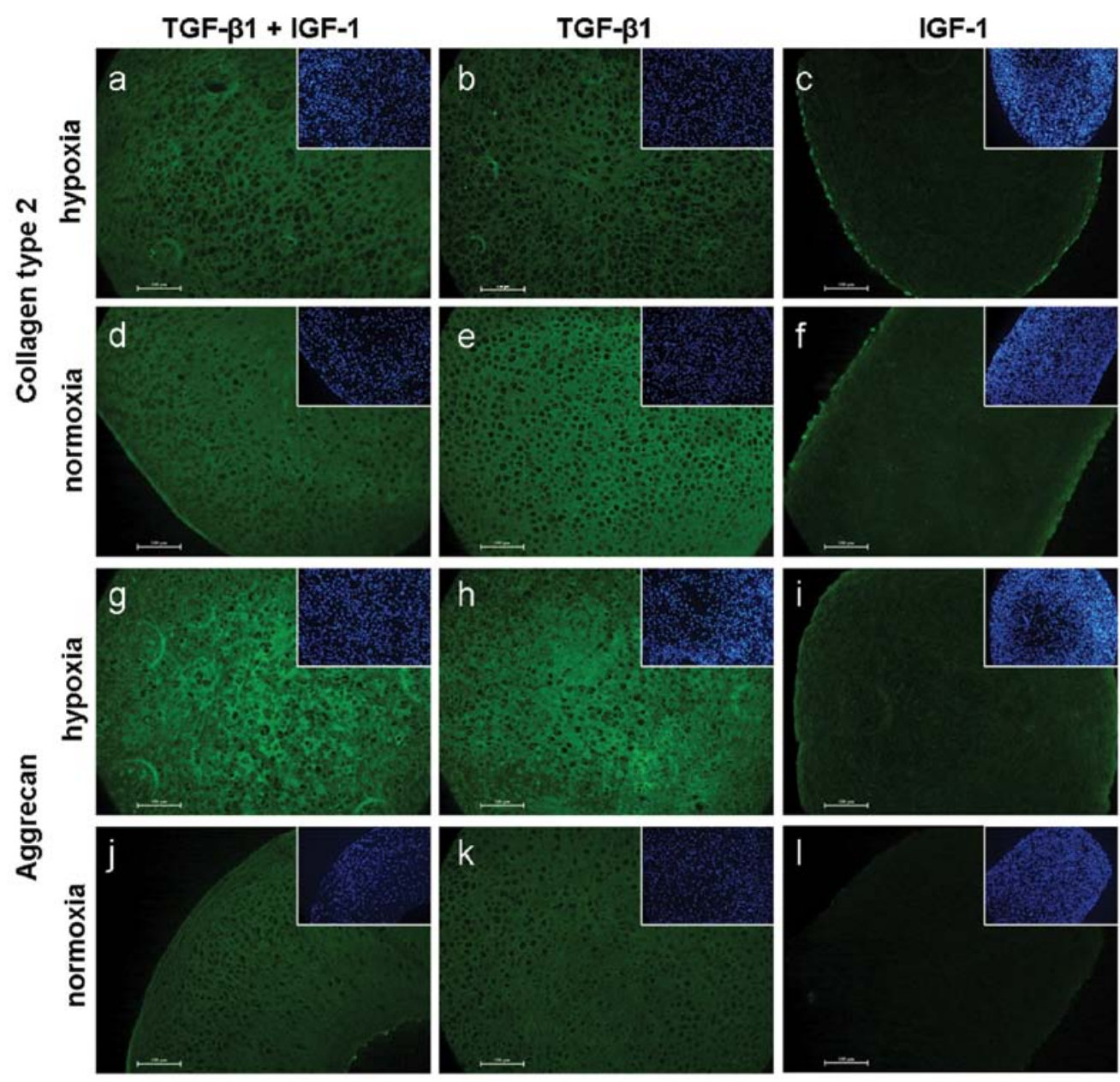

Figure 4. Immunhistological stainings of (a-f) collagen type 2 and (g-l) aggrecan of human chondrocytes cultivated in pellet cultures with TGF- $\beta 1$, IGF-1 and the combination of both after 14 and 35 days. Photographs were taken with a Nikon Eclipse TS100 microscope using an objective with a 20-fold magnification (bar, $500 \mu \mathrm{m} ; \mathrm{n} \geq 2$ ).
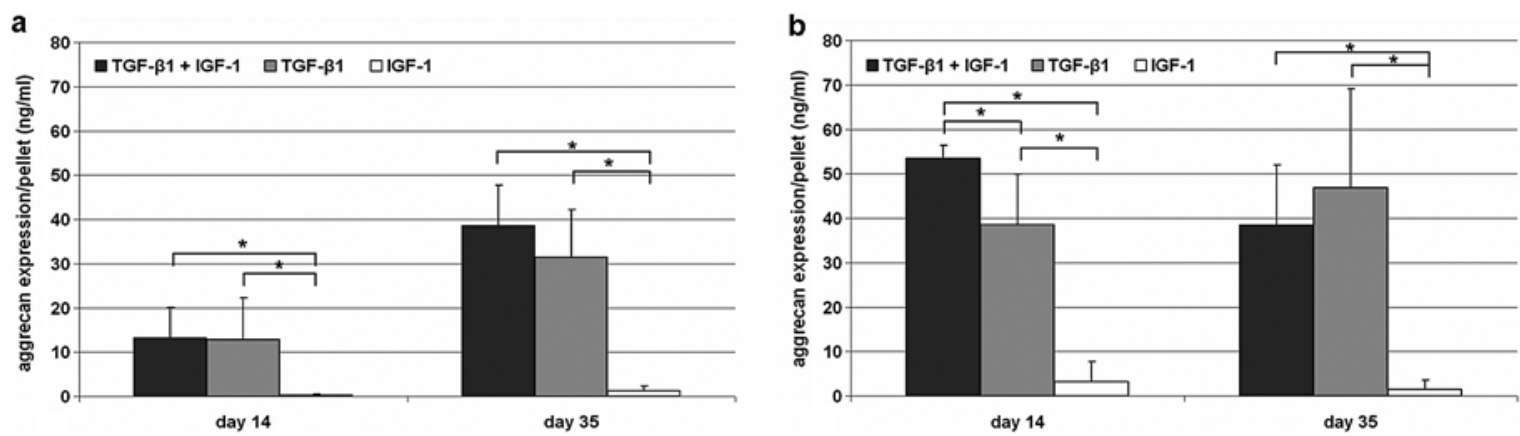

Figure 5. Aggrecan synthesis of human chondrocyte pellet cultures under (a) hypoxic and (b) normoxic culture conditions with different stimulating combinations ( $\mathrm{n}=4$, in duplicates). Data are presented as means \pm standard deviation. Comparison between the three growth factor combinations was conducted with the Kruskal-Wallis test for independent samples or the Mann-Whitney test for paired samples. Comparison between the both culture conditions was conducted with the Mann-Whitney test; ${ }^{*} \mathrm{P}<0.05$. Statistical significance between the two time points was calculated with the Wilcoxon test.

differences $(\mathrm{P}=0.029)$ could be determined for the stimulating experiments on both time points (Fig. 5).

Staining of aggrecan resulted in visible differences between the culture conditions. A brighter positive staining of TGF- $\beta 1 \pm$ IGF-1 treated pellets was observed under hypoxia. Furthermore, a positive staining of aggrecan was determined after IGF-1 treatment in hypoxic cultivated pellets only (Fig. 4g-1).

\section{Discussion}

The aim of this study was to analyse the effect of TGF- $\beta 1$, IGF-1 or the combination of both growth factors on the proliferation and synthesis rate of collagen type 1 and type 2 as well as aggrecan of human chondrocytes cultivated as pellet cultures under differing oxygen contents. Several studies about the chondrogenic potential of several cell types, e.g. 
MSC from adipose tissue or bone marrow, in pellet cultures were performed but only limited studies have addressed this aspect by using chondrocytes from human articular cartilage. In a previous study, we could show that cells isolated from hyaline cartilage tissue express high levels of MSC-associated markers which play an important role during chondrogenesis (14). Additionally, pellet cultures allow cell to cell interactions which are similar to those in pre-cartilage condensation found during embryonic development (16). Based on these findings, we hypothesised that the addition of chondrogenic growth factors could influence re-differentiation processes of cells in a 3D environment.

After the treatment with different chondrogenic growth factors under hypoxia and normoxia, differences in DNA contents within the pellets could be observed. Although we used the same initial cell number, a reduced DNA content could be observed after 14 days under normoxia compared to hypoxic conditions. After 35 days of incubation under normoxia we could show an increase in DNA content after treatment with TGF- $\beta 1$ alone or in combination with IGF-1. One possible explanation could be the addition of TGF- $\beta 1$ which is known to rise the proliferation of mesenchymal stem cells during chondrogenesis (11). Nevertheless, the values of DNA content under hypoxia could not be achieved after cultivation under normoxic conditions. Furthermore, the single treatment with IGF-1 led to a decrease in DNA content during cultivation. We suggest that under normoxia, differences in oxygen concentrations predominate between cells in the periphery and in the centre of the pellet. As a consequence, cells are exposed to an increased oxidative stress, which results in a higher apoptosis rate. In this context, the first results indicated an enhanced apoptosis of cells after 14 days of incubation under normoxia which has to be examined in a subsequent study. Additionally, normoxia resulted in an enrichment of reactive oxygen species (ROS) which lead to a reduced activity of especially IGF-1 (2). In contrast, under hypoxic conditions the hypoxic-inducible transcription factor (HIFs) proteins are permanently expressed within chondrocytes (17) which regulate differentiation of cells. Bohensky et al (18) showed that HIF-2 $\alpha$ could prevent apoptosis of chondrocytes, limiting ROS production. As our results demonstrate, chondrocyte-pellets incubated under hypoxia showed less evidence of cell death because of a stable DNA content. Additionally, the chosen culture conditions of $5 \%$ oxygen is more physiological for chondrogenic cells because in articular cartilage the oxygen concentration is estimated to range between 1 and 5\% (17).

For the synthesis of specific ECM components, differences in the expression between hypoxia and normoxia as well as differences between growth factors could be observed. For the experiments we used TGF- $\beta 1$ and IGF-1 because both growth factors play an important role for ECM formation $(12,19,20)$. Under both culture conditions a reduced synthesis rate of pro-collagen type 1 could be shown after stimulation with the three growth factor compositions. For IGF-1 treated pellets a reduced synthesis rate of pro-collagen type 1 compared to TGF- $\beta 1$ as well as TGF- $\beta 1 /$ IGF- 1 stimulated ones could be shown at both time points. The difference between the combinations was significant on Day 35. As a consequence, a more positive effect of IGF-1 on re-differentiation of chondrocytes is observed compared to TGF- $\beta 1$.
In general, under normoxia we detected an enhanced synthesis rate of pro-collagen type 2 and aggrecan after the stimulation with all growth factor combinations compared to hypoxic culture conditions. This was not unexpected because we used chondrocytes isolated from osteoarthritic cartilage. It is well known, that these cells are characterised by an increased expression of collagen type 2 and other matrix components as a response to restore ECM (21).

Additionally, after 35 days of incubation the TGF- $\beta 1$ and/or IGF-1 stimulation led to an increased concentration of pro-collagen type 2 under normoxia. These results were in correspondence with the DNA data because proliferating chondrocytes synthesise collagen type 2 (22). For aggrecan, we could show an accumulation after TGF- $\beta 1$ treatment under normoxia whereas a reduced expression was determined after TGF- $\beta 1 /$ IGF-1 as well as IGF-1 treatment.

Under hypoxic culture conditions expression of pro-collagen type 2 and aggrecan increased during the stimulation with TGF- $\beta 1 /$ IGF- 1 . In contrast to normoxia, a stable pro-collagen type 2 concentration after single treatment with IGF-1 and TGF- $\beta 1$ was determined. Furthermore, IGF- 1 stimulation increased aggrecan synthesis. Although IGF-1 effects on the accumulation of ECM components were significantly reduced compared to TGF- $\beta 1 /$ IGF-1, IGF-1 seemed to be more efficient. Under hypoxia, expression of collagen type 2 and aggrecan are mediated by HIF- $1 \alpha$ which is the survival factor in chondrocytes (23). Additionally, growth factors like IGF-1 also influence HIF-1 activity positively (21) which could be an explanation for superior results of aggrecan accumulation after IGF-1 treatments under hypoxia.

Nevertheless, the IGF-1 effects on the ECM component expression were clearly reduced under normoxia as well as under hypoxia compared to TGF- $\beta 1$ treatment. These results are in accordance to Chubinskaya et al (9), who did not verify matrix accumulation after single treatment of IGF-1 in alginate beads. One reason for these effects could be the cell source used. For our experiments, we isolated cells from osteoarthritic cartilage. Martin et al (24) reported an enhanced proportion of the IGF-binding protein 3 as well as fibronectin in OA cartilage. Colocalisation of both components in the ECM resulted in controlling of IGF-1 because of complex formation. For this reason, the inhibitory effect of IGFBP 3 is retained (8) and IGF-1 could not be released. This may discredit the applicability of IGFBP pathways as targets for cartilage repair (25). Gonzalez et al (25) emphasised the impact of a short-term TGF- $\beta 1$ stimulation to inhibit IGFBP production. As a result, applied IGF-1 could not only be stored in the ECM but also bound on IGF-receptors to activate the intracellular pathway to stimulate collagen type 2 and proteoglycan synthesis. Under hypoxia an additive effect on matrix deposition after stimulation with TGF- $\beta 1 /$ IGF- 1 was detected. We assume a positive influence of TGF- $\beta 1$ on IGF-1 when using the combination of both under hypoxic culture conditions.

In summary, treatment with IGF- 1 and TGF- $\beta 1$ influences the re-differentiation of chondrocytes with respect to the accumulation of hyaline ECM compound. Nevertheless, our tests revealed differences between cultivation conditions concerning the oxygen content. While under normoxic conditions TGF- $\beta 1$ alone leads to a positive impact of the expression of hyaline cartilage-specific ECM components, an additive 
effect of both growth factors was found under hypoxic conditions. IGF-1 treatment under hypoxia was more efficient than under normoxia. Thereby, our hypothesis concerning oxygen content reduction could be confirmed by the data of DNA concentration and ECM accumulation, i.e. under hypoxia ROS enrichment could be limited resulting in a positive impact for IGF-1.

Hence, a natural 3D environment in combination with chondrogenic growth factors as well as reduced oxygen contents could facilitate cell survival and chondrogenic re-differentiation. However, in further studies it is recommended to analyse the effects of growth factors in combination with normoxia or hypoxia with chondrocytes derived from donors without osteoarthritis. Detailed knowledge about the cell interactions in the context of environment conditions could lead to targeted differentiation of cartilage-derived cells into hyaline cartilage as a prerequisite for the development of new therapeutic strategies in cartilage repair.

\section{Acknowledgements}

The authors gratefully thank the European Union and the Ministry of Economic Affairs, Employment and Tourism of Mecklenburg-Vorpommern for financial support within the research project 'Syntero'. We thank Mrs. Frauke Winzer (Department of Anatomy) for her technical support with immunohistochemical preparation.

\section{References}

1. Farr J, Cole B, Dhawan A, Kercher J and Sherman S: Clinical cartilage restoration: evolution and overview. Clin Orthop Relat Res 469: 2696-2705, 2011.

2. Loeser RF: Aging and osteoarthritis: the role of chondrocyte senescence and aging changes in the cartilage matrix. Osteoarthritis Cartilage 17: 971-979, 2009.

3. Giovannini S, Diaz-Romero J, Aigner T, Heini P, Mainil-Varlet P and Nesic D: Micromass co-culture of human articular chondrocytes and human bone marrow mesenchymal stem cells to investigate stable neocartilage tissue formation in vitro. Eur Cell Mater 20: 245-259, 2010

4. Peterson L, Vasiliadis HS, Brittberg $M$ and Lindahl A: Autologous chondrocyte implantation: a long-term follow-up. Am J Sports Med 38:1117-1124, 2010.

5. Stokes DG, Liu G, Dharmavaram R, Hawkins D, PieraVelazquez S and Jimenez SA: Regulation of type-II collagen gene expression during human chondrocyte de-differentiation and recovery of chondrocyte-specific phenotype in culture involves Sry-type high-mobility-group box (SOX) transcription factors. Biochem J 360: 461-470, 2001.

6. Benya PD and Shaffer JD: Dedifferentiated chondrocytes reexpress the differentiated collagen phenotype when cultured in agarose gels. Cell 30: 215-224, 1982.

7. Bonaventure J, Kadhom N, Cohen-Solal L, Ng KH, Bourguignon J, Lasselin $\mathrm{C}$ and Freisinger P: Reexpression of cartilage-specific genes by dedifferentiated human articular chondrocytes cultured in alginate beads. Exp Cell Res 212: 97-104, 1994.

8. van der Kraan PM, Buma P, van KT and van den Berg WB: Interaction of chondrocytes, extracellular matrix and growth factors: relevance for articular cartilage tissue engineering. Osteoarthritis Cartilage 10: 631-637, 2002.
9. Chubinskaya S, Hakimiyan A, Pacione C, Yanke A, Rappoport L, Aigner T, Rueger DC and Loeser RF: Synergistic effect of IGF-1 and OP-1 on matrix formation by normal and OA chondrocytes cultured in alginate beads. Osteoarthritis Cartilage 15: 421-430, 2007.

10. Giannoni P and Cancedda R: Articular chondrocyte culturing for cell-based cartilage repair: needs and perspectives. Cells Tissues Organs 184: 1-15, 2006.

11. Fukumoto T, Sperling JW, Sanyal A, Fitzsimmons JS, Reinholz GG, Conover CA and O'Driscoll SW: Combined effects of insulin-like growth factor-1 and transforming growth factorbeta1 on periosteal mesenchymal cells during chondrogenesis in vitro. Osteoarthritis Cartilage 11: 55-64, 2003.

12. Yaeger PC, Masi TL, de Ortiz JL, Binette F, Tubo R and McPherson JM: Synergistic action of transforming growth factor-beta and insulin-like growth factor-I induces expression of type II collagen and aggrecan genes in adult human articular chondrocytes. Exp Cell Res 237: 318-325, 1997.

13. van Osch GJ, van der Veen SW, Buma P and VerwoerdVerhoef HL: Effect of transforming growth factor-beta on proteoglycan synthesis by chondrocytes in relation to differentiation stage and the presence of pericellular matrix. Matrix Biol 17: 413-424, 1998.

14. Jonitz A, Lochner K, Peters K, Salamon A, Pasold J, MuellerHilke B, Hansmann D and Bader R: Differentiation capacity of human chondrocytes embedded in alginate matrix. Connect Tissue Res 52: 503-511, 2011.

15. Guerne PA, Blanco F, Kaelin A, Desgeorges A and Lotz M: Growth factor responsiveness of human articular chondrocytes in aging and development. Arthritis Rheum 38: 960-968, 1995.

16. Zhang L, Su P, Xu C, Yang J, Yu W and Huang D: Chondrogenic differentiation of human mesenchymal stem cells: a comparison between micromass and pellet culture systems. Biotechnol Lett 32: 1339-1346, 2010.

17. Lafont JE: Lack of oxygen in articular cartilage: consequences for chondrocyte biology. Int J Exp Pathol 91: 99-106, 2010.

18. Bohensky J, Terkhorn SP, Freeman TA, Adams CS, Garcia JA, Shapiro IM and Srinivas V: Regulation of autophagy in human and murine cartilage: hypoxia-inducible factor 2 suppresses chondrocyte autophagy. Arthritis Rheum 60: 1406-1415, 2009.

19. Tyler JA: Insulin-like growth factor 1 can decrease degradation and promote synthesis of proteoglycan in cartilage exposed to cytokines. Biochem J 260: 543-548, 1989.

20. Morales TI: The insulin-like growth factor binding proteins in uncultured human cartilage: increases in insulin-like growth factor binding protein 3 during osteoarthritis. Arthritis Rheum 46: 2358-2367, 2002.

21. Pfander D and Gelse K: Hypoxia and osteoarthritis: how chondrocytes survive hypoxic environments. Curr Opin Rheumatol 19: 457-462, 2007.

22. Schipani E: Posttranslational modifications of collagens as targets of hypoxia and Hif-1alpha in endochondral bone development. Ann NY Acad Sci 1192: 317-321, 2010.

23. Duval E, Leclercq S, Elissalde JM, Demoor M, Galera P and Boumediene K: Hypoxia-inducible factor lalpha inhibits the fibroblast-like markers type I and type II collagen during hypoxia-induced chondrocyte redifferentiation: hypoxia not only induces type II collagen and aggrecan, but it also inhibits type I and type II collagen in the hypoxia-inducible factor 1alphadependent redifferentiation of chondrocytes. Arthritis Rheum 60: 3038-3048, 2009.

24. Martin JA, Miller BA, Scherb MB, Lembke LA and Buckwalter JA: Co-localization of insulin-like growth factor binding protein 3 and fibronectin in human articular cartilage. Osteoarthritis Cartilage 10: 556-563, 2002.

25. Gonzalez C, Auw Yang KG, Schwab JH, Fitzsimmons JS, Reinholz MM, Resch ZT, Bale LK, Clemens VR, Conover CA, O'Driscoll SW and Reinholz GG: Transforming growth factorbeta1 modulates insulin-like growth factor binding protein-4 expression and proteolysis in cultured periosteal explants. Growth Horm IGF Res 20: 81-86, 2010. 